\title{
Thermodynamic Optimization of Critical Metals Processing and Recovery: Part I
}

\author{
CHUKWUNWIKE O. ILOEJE (i), ${ }^{1,4}$ FISEHA TESFAYE, ${ }^{2,5}$ \\ and ALEXANDRA E. ANDERSON ${ }^{3,6}$ \\ 1.-Energy Systems Division, Argonne National Laboratory, 9700 South Cass Avenue, \\ Lemont, IL 60439-6903, USA. 2.-Johan Gadolin Process Chemistry Centre, Ảbo Akademi \\ University, Piispankatu 8, 20500 Turku, Finland. 3.-Gopher Resource, 6505 Jewel Ave, \\ Tampa, FL, USA. 4.-e-mail: ciloeje@anl.gov. 5.—e-mail: fiseha.tesfaye@abo.fi. \\ 6.—e-mail: allieanderson0@gmail.com
}

Several metals used in high-tech applications are predominantly produced as byproducts in the extraction processing of certain primary metals, such as copper and zinc. For instance, $>90 \%$ global supply of selenium and tellurium are produced as byproducts of copper smelting plants. In base metals smelting processes, these byproduct metals, including precious metals, are distributed in the slag and metal phases as trace elements and mainly recovered from anode slimes, which is a residual byproduct formed during electrolytic refining, as shown in Fig. 1.

Today, various smelting processes incorporate the processing of secondary resources, such as waste electrical and electronic equipment (e-waste). The increasing amount and complexity of e-waste introduces new and additional valuable metals into conventional smelting circuits. For the most efficient recovery of these valuable metals-and to define optimal processing parameters-fundamental knowledge regarding the behavior and impact of different (trace) elements and process chemistry is essential. ${ }^{1}$

These trends motivate the need to develop systematic thermodynamic optimization theory and techniques, and to conduct detailed material processing analysis to identify optimal parametric relationships, process conditions, and processing strategies that increase resource recovery, improve energy efficiency and reduce waste generation in metals production from primary and secondary sources. This highlights the value proposition of fundamental research, such as determining the phase transition behavior of superalloy systems

(Received December 7, 2020; accepted December 9, 2020; published online January 18, 2021) and understanding the effects of composition and processing conditions on material properties and process yield efficiencies.

Recently, JOM advisors of the Process Technology and Modeling Committee and Recycling and Environmental Technologies Committee of TMS organized a special topic on Thermodynamic Modeling of Sustainable Non-Ferrous Metals Production, ${ }^{2}$ which incorporated articles with a focus on hightemperature experimental investigations and thermodynamic modeling, specifically those pertaining to non-ferrous metallurgical processes for recovery of metals like $\mathrm{Pb}, \mathrm{Ti}$, and $\mathrm{Au}$.

This special, two-part topic extends this series by bringing focus to the "Thermodynamic Optimization of Critical Metals Processing and Recovery," which invited manuscripts that address topics in generalized theory, solution algorithms, specific materials processing units or whole production processes, as well as those that integrate experiments. This first part of the two-part topic comprises articles that describe experimental and modeling work that investigate material property evolution, optimal processing parameters, and thermodynamic optimization approaches both for superalloy development and extractive metallurgy processes. After peer review by several experts in the field, six original research articles were approved for publication.

Two articles focused on the thermodynamic optimization of $\mathrm{Fe}$-containing superalloys are presented within this special topic The first of these articles, "Precipitation Behavior of Carbide and its Effect on the Mechanical Property of a Novel $\mathrm{Fe}_{60} \mathrm{Co}_{10} \mathrm{Cr}_{10}$ $\mathrm{Ni}_{10} \mathrm{Mo}_{5} \mathrm{~V}_{5}$ Medium-Entropy Alloy" by Hebin Wang et al., explores the effect of aging parameters, temperature, and time on the nucleation mechanism of carbide precipitates, and the resulting 


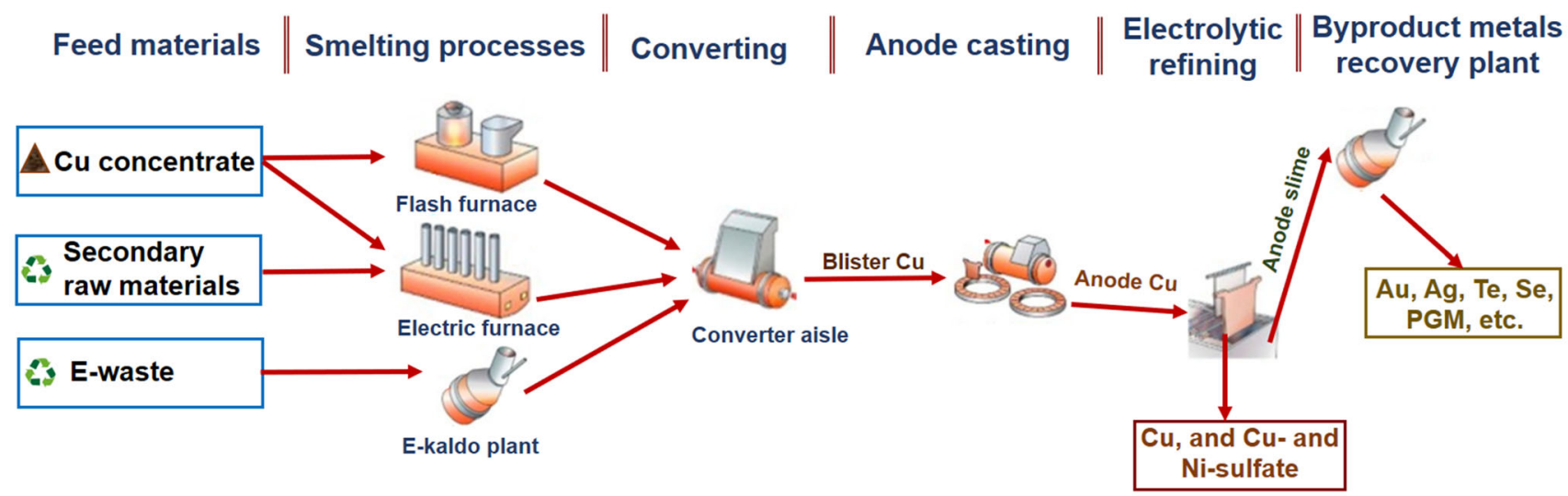

Fig. 1. A simplified schematic process flow sheet of the copper smelter plant of Boliden Rönnskär in Skelleftehamn, Sweden, integrating e-waste processing and the byproduct metals recovery plant; adapted and modified from Ref. 3

microstructure and mechanical property evolution of the alloy, with the objective of improving material strength and stability. Key outcomes show a hardening temperature range of $400^{\circ} \mathrm{C}$ to $1000^{\circ} \mathrm{C}$-with an optimal hardness observed around $800^{\circ} \mathrm{C}$ - and identifies precipitation and solid solution strengthening as the primary property evolution mechanisms.

In the second of these two articles, "Thermodynamic Assessment of Liquid Fe-Ni-C Alloy Using Modified Quasichemical Model," Min-Kyu Paek et al. apply a modified quasi-chemical model, which describes the asymmetry of $\mathrm{Fe}-\mathrm{Ni}$ and Fe-Ni-C liquid solution properties, for the thermodynamic optimization of $\mathrm{Fe}-\mathrm{Ni}$ and $\mathrm{Fe}-\mathrm{Ni}-\mathrm{C}$ systems relevant to high nickel-containing alloys. The study reproduced the extrema partial properties of $\mathrm{C}$ in the ternary Fe-Ni-C system with a single adjustable ternary parameter, and showed that the solubility limits and maximum partial mixing enthalpy of $\mathrm{C}$ in the ternary Fe-Ni-C system are strongly correlated with the short-range ordering composition of the binary Fe-Ni liquid solution.

The last four articles included in this special topic deal with various extractive metallurgy processes in the gold, copper, and steelmaking industries. Jun Gil Yang et al. present a study entitled "Gold Solubility in $\mathrm{CaO}-\mathrm{SiO}_{2}-\mathrm{Al}_{2} \mathrm{O}_{3}-\mathrm{Fe}_{2} \mathrm{O}_{3}$ Slags," which investigates the high-temperature dissolution behavior of gold as a function of slag composition, expressed in terms of relevant basicity indices. The study results showed that gold solubility in the slag system increased with increasing slag basicity, with a strong dependence on the $\mathrm{CaO} / \mathrm{SiO}_{2}$ ratio, optical basicity, and $\mathrm{CaO}$ activity, and negligible influence from the presence of $\mathrm{Fe}_{2} \mathrm{O}_{3}$ and $\mathrm{Al}_{2} \mathrm{O}_{3}$. These results inform system designs that maximize gold recovery (minimize gold loss to slag phase) during pyrometallurgical processing of gold-containing e-waste.

The next two articles focus on the recovery of metals from the pyrometallurgical processing of copper. Firstly, in their article "A Study of Selenium and Tellurium Distribution Behavior in a Simulated
Copper Matte Flash Converting Process," Yu et al. performs an experimental study aimed at a better understanding of selenium and tellurium phase distribution behaviors within copper flash smelting materials. The deportment of selenium and tellurium to the oxide, matte, and metal phases was examined as a function of impurity concentration, temperature, and flux additions. It is the authors' hope that the data generated from this work will be used to enhance the recovery efficiencies of these critical elements within the copper industry. Secondly, the article entitled "Investigation of solidstate carbothermal reduction of fayalite without and with added metallic iron" by Hongyang Wang et al. describes the carbothermal reduction kinetics of iron-bearing fayalite, a common constituent of waste slag from pyrometallurgical processing of copper, as a function of metallic iron addition, time, and temperature. A comparison of specimens produced with and without the addition of metallic iron was also conducted using XRD and SEM-EDS to better understand the reaction mechanism involved with the use of metallic iron as a reactant.

Lastly, in the fourth extractive related article, "Evaluation and Modeling of Scrap Utilization in the Steelmaking Process," Ming Gao et al. assess the performance of the steel scrap melting process using various statistical methods. The models developed through this study examined the influence of several process parameters, which included scrap properties as well as furnace operating conditions, on the rate of melting and the overall mass transfer coefficient. The analysis showed that mixing and bath temperature had the greatest impact on scrap melting, and several findings from the statistical models were used to recommend methods for improving the efficiency of the overall process.

The papers published in this issue should be of interest to a broad readership including those planning to minimize environmental footprints while promoting sustainable production of the critical metals meeting future demands. All titles and authors of the articles published under the topic 
"Thermodynamic Optimization of Critical Metals Processing and Recovery: Part I" in the February 2021 issue (vol. 73, no. 2) of JOM are listed below. The articles can be accessed fully via the journal's page at: http://link.springer.com/journal/11837/73/2/ page/1.

- "Precipitation Behavior of Carbide and Its Effect on the Mechanical Property of a Novel Fe60Co10Cr10Ni10Mo5V5 Medium-Entropy Alloy" by H. Wang, D. Hong, L. Hou, P. Ou, L. Shen, Y. Wang, Z. Wang, and $\mathrm{H}$. Zhao.

- "Gold Solubility in $\mathrm{CaO}-\mathrm{SiO}_{2}-\mathrm{Al}_{2} \mathrm{O}_{3}-\mathrm{Fe}_{2} \mathrm{O}_{3}$ Slags" by J.G. Yang, J.H. Park, J.Y. Kang, H.S. Park, and J. Park.

- "Thermodynamic Assessment of Liquid Fe-Ni-C Alloy Using Modified Quasichemical Model" by M. Paek, J. Jeon, M. Paliwal, A. Biswas, D. Lindberg, and J.-J. Pak.

- "A Study of Selenium and Tellurium Distribution Behavior in a Simulated Copper Matte Flash Converting Process" by F. Yu, Z. Liu, F. Ye, L. Xia, and A. Jokilaakso.

- "Evaluation and Modeling of Scrap Utilization in the Steelmaking Process" by M. Gao, J.T. Gao, Y. Zhang, and S.F. Yang.

- "Investigation of Solid-State Carbothermal Reduction of Fayalite without and with Added Metallic Iron" by H. Wang, L. Shen, H. Bao, W. Zhang, X. Zhang, L. Luo, and S. Song.

\section{ACKNOWLEDGEMENTS}

The organizers of this special topic would like to thank all authors, co-authors, and reviewers who have contributed to the successful publication of this issue. We are convinced that your expertise has resulted in an excellent issue of $J O M$ on this timely topic. We acknowledge support from UChicago
Argonne LLC, Operator of Argonne National Laboratory ("Argonne"). Argonne National Laboratory's work was supported by the U.S. Department of Energy, Office of Energy Efficiency and Renewable Energy (EERE), under Contract DE-AC0206CH11357. The U.S. Government retains for itself, and others acting on its behalf, a paid-up nonexclusive, irrevocable worldwide license in said article to reproduce, prepare derivative works, distribute copies to the public, and perform publicly and display publicly, by or on behalf of the Government. The Department of Energy will provide public access to these results of federally sponsored research in accordance with the DOE Public Access Plan. The authors are also grateful to the Academy of Finland (Decision Number 311537) for financial support as part of the activities of the Johan Gadolin Process Chemistry Centre at Åbo Akademi University.

\section{CONFLICT OF INTEREST}

The authors declare that they have no conflict of interest.

\section{REFERENCES}

1. L. Klemettinen, K. Avarmaa, and D. Sukhomlinov, et al., $S N$ Appl. Sci. 2, 337 (2020). https://doi.org/10.1007/s42452-0202137-1.

2. F. Tesfaye, A.E. Anderson, and M. Zhang, JOM 72, 3176 (2020). https://doi.org/10.1007/s11837-020-04283-5.

3. F. Tesfaye, D. Lindberg, and J. Hamuyuni, Valuable metals and energy recovery from electronic waste streams.Energy Technology 2017, ed. L. Zhang, et al.The Minerals, Metals \& Materials Series, (Cham: Springer, 2017), https://doi.org/10. 1007/978-3-319-52192-3_11.

Publisher's Note Springer Nature remains neutral with regard to jurisdictional claims in published maps and institutional affiliations. 